\title{
Aporte de la geografía al análisis de la gestión de crisis ${ }^{1}$
}

JÉRÉMY ROBERT

> Instituto Francés de Estudios Andinos, IFEA, Francia. Pontificia Universidad Católica del Perú, Perú. jrobert@pucp.pe

Universidad de Valparaíso

Facultad de Arquitectura

Revista Márgenes

Espacio Arte Sociedad

Aporte de la geografía al análisis de la gestión de crisis

Diciembre 2014 Vol. $11 \mathrm{~N}^{\circ} 15$

Páginas 32 a 38

ISSN elec. 0719-4463

ISSN imp. 0718-4034

Recepción: Junio 2014

Aceptación: Octubre 2014

\section{RESUMEN}

Las crisis en el medio urbano representan un desafío mayor para las sociedades contemporáneas. Al cuestionar los esquemas de pensamiento y los modos de respuestas, estas recuerdan constantemente la necesidad de producción de conocimientos. Tratada por numerosas disciplinas en ciencias sociales, la gestión de las crisis ha sido abordada rara vez por los geógrafos, aún cuando las dimensiones espaciales y territoriales de las crisis son innegables. Dentro de este contexto, el artículo propone fijar los hitos de una geografía de la gestión de crisis. Luego de una presentación de las especificidades de las crisis en el medio urbano, este rastrea los diferentes acercamientos de la cuestión dados por los geógrafos y propone un enfoque original que aborda frontalmente las dimensiones espaciales y territoriales del manejo de las situaciones de crisis. La reflexión se apoya en gran parte sobre el trabajo de tesis realizado en Lima en 2008 y 2012, que propone una geografía de la gestión de crisis a partir de la accesibilidad a los cuidados de la salud en situación de emergencia y de la vulnerabilidad del territorio (Robert, 2012).

PALABRAS CLAVES

catástrofes, gestión de crisis, geografía, vulnerabilidad urbana, riesgo, Lima

\section{Contribution of geography to the analysis of the crisis management ABSTRACT}

Urban crises represent a major challenge for contemporary societies. Disasters are a constant reminder of the necessity of production of knowledge by calling into question current frameworks and modalities of response. Handled by numerous disciplines of social science, the question of these crises has rarely been studied by geographers, even when spatial and territorial dimensions of the crises are undeniable. In this context, the article suggests establishing milestones for crisis management geography. After a presentation of the specific crises in urban areas, this article redraws different approaches to the question by geographers and proposes an original approach that directly tackles the spatial and territorial dimensions of crisis management. The reflection leans largely on a PhD research project based in Lima in 2008 and 2012, which proposes a crisis management geography based on the accessibility to emergency healthcare and territorial vulnerability (Robert, 2012).

KEYWORDS

disasters, crisis management, geography, urban vulnerability, risk, Lima

\section{La gestion de crise: un point de vue de géographe RÉSUMÉ}

Les crises en milieu urbain représentent un défi majeur pour les sociétés contemporaines. Remettant en question les schémas de pensée et les modes de réponses, elles rappellent constamment la nécessité de production de connaissances. Traitée par de nombreuses disciplines en sciences sociales, la question des crises n'a que rarement été abordée par les géographes, alors même que les dimensions spatiales et territoriales des crises sont indéniables. Dans ce contexte, l'article propose de poser les jalons d'une géographie de la gestion de crise. Après une présentation des spécificités des crises en milieu urbain, il retrace les différentes approches de la question par les géographes et propose une démarche originale qui aborde frontalement les dimensions spatiales et territoriales de la gestion des situations de crise. La réflexion s'appuie en grande partie sur le travail de thèse réalisé à Lima en 2008 et 2012, qui propose une géographie de la gestion de crise à partir de l'accessibilité aux soins en situation d'urgence et de la vulnérabilité du territoire MOTS CLÉS

catastrophes, gestion de crise, géographie, vulnérabilité urbaine, risque, Lima 
Las crisis que afectan a las grandes aglomeraciones son una de las preocupaciones mayores de nuestras sociedades: primero, porque más de la mitad de la población mundial es hoy en día urbana, luego porque las crisis no parecen tener límites, ya sea en términos de víctimas o de costos. Estas crisis plantean una multitud de problemas, algunos más bien de orden logístico y técnico, otros institucionales y sociales.

Mientras la geografía de riesgos dispone de bases sólidas, la cuestión de las crisis no ha sido sino rara vez abordada por los geógrafos. Sin embargo, las dimensiones espaciales que caracterizan las situaciones de crisis son innegables. Estas se traducen en lugares, desplazamientos y flujos extraordinarios, provocados por los daños que afectan a las viviendas, a las redes vitales o a las zonas de actividades. Algunos espacios residenciales deben ser abandonados (al menos parcialmente), se paralizan zonas de actividad laboral, los flujos usuales relacionados con el trabajo, el entretenimiento o la educación son reemplazados por otros, determinados por la emergencia. La gestión misma de la crisis, destinada a aportar auxilio a la población y a volver a poner en servicio lugares, infraestructuras y redes del sistema urbano, va a acarrear la intervención de nuevos actores, generar formas de acción excepcionales y objetivos específicos de la acción pública. La pertinencia de los territorios de gestión habituales es puesta en tela de juicio, aparecen nuevos lugares estratégicos y se ve emerger nuevos actores y espacios de intervención.

Estos aspectos son evocados a veces en los análisis y los retornos de experiencia de gestión de crisis pero rara vez son objeto de investigaciones específicas. En efecto, si bien existen investigaciones en ciencias sociales sobre la gestión de crisis, una geografía de los espacios y territorios de la gestión de crisis está todavía por construirse. A partir de los retornos de experiencias, intentaremos caracterizar en un primer momento los grandes desafíos de la gestión de crisis en el medio urbano. Después de recordar el contexto de la investigación sobre las crisis, veremos cómo es tratado este tema por los geógrafos. Finalmente se discutirá el interés de una investigación geográfica que aborda frontalmente la cuestión de la gestión de crisis.

\section{ESPECIFICIDAD DE LAS GRANDES CRISIS EN MEDIO URBANO}

Basándonos principalmente en tres crisis urbanas mayores provocadas por el sismo de México D. F. en 1985, el huracán Katrina en Nueva Orleans en 2005 y el sismo de Puerto Príncipe (Haití) en 2010, subrayaremos aquí cinco características de las grandes crisis urbanas.

\section{La superación de los órdenes de magnitud}

La ciudad es por esencia un lugar de concentración de población. Las crisis en el medio urbano provocan una demanda masiva e inusual, los órdenes de magnitud explosionan: 2 millones de personas sin hogar en México D. F. en 1985, 250 mil evacuados de la ciudad de Nueva Orleans y 60 mil personas permanecieron bloqueadas por la inundación que siguió al huracán Katrina en 2005, más de 200 mil muertos y 300 mil heridos en Puerto Príncipe en 2010. Al mismo tiempo, los recursos que permiten responder a ello se encuentran ampliamente afectados, y si es que no están totalmente fuera de servicio, funcionan de manera degradada.
La ciudad es también una concentración de elementos mayores del funcionamiento de las sociedades modernas. La urbanización se traduce por el incremento de la producción de riquezas por m2. Esta concentración de bienes explica la amplitud y el incremento incesante de los montos de las grandes catástrofes. Las pérdidas globales ocasionadas por Katrina, estimadas a 200 mil millones de dólares, constituyeron un récord hoy superado por la catástrofe del Japón en marzo de 2011, estimadas en 300 mil millones de dólares. Estos costos tienen que ser puestos dentro de los contextos nacionales, en particular en los países del sur. El monto de los daños generados por el sismo de Haití representa más del 50\% de su PIB anual.

\section{Imbricaciones de las redes técnicas}

La segunda especificidad de las crisis en el medio urbano atañe al daño de las infraestructuras vitales y los efectos en cadena que afectan a las grandes redes indispensables para el funcionamiento urbano. Hoy en día estos tienden a multiplicarse y a imbricarse de manera cada vez más densa, traduciendo una complexificación creciente de su funcionamiento e interdependencias cada vez más marcadas (Michel-Kerjan, 2003; Dubois-Maury et Chaline, 2004).

Cuando ocurren grandes crisis, las redes de telecomunicaciones, de abastecimiento en agua y en energía, etc., son sistemáticamente deficientes, ocasionando efectos en cadena y perturbaciones mayores. El daño de un elemento puntual puede tener repercusiones sobre el conjunto de un servicio: en México D. F., la destrucción del edificio que albergaba las infraestructuras claves de las telecomunicaciones interrumpió el servicio a escala metropolitana y nacional. De igual manera, fueron los cortes de agua y de electricidad los que obligaron a los hospitales de Nueva Orleans a evacuar a sus pacientes, y no necesariamente los daños directos causados por el huracán y la inundación que lo siguió. Como consecuencia de estas imbricaciones e interdependencias, se constata que más allá de la naturaleza y de la intensidad del evento exterior perturbador, cada deficiencia de estas redes contribuye a agravar la crisis.

\section{Multiplicidad de los actores}

La multiplicidad de los actores en presencia es otra fuente de complejidad. Ya en tiempos normales, las ciudades son teatro de la actuación de actores múltiples y de conflictos. En situación de crisis, el número de actores se acrecienta y todos los conflictos resurgen, se enfatizan y se amplifican. En México D. F., fueron muy marcados tanto la intervención de las organizaciones locales y de actores privados ya sea para apoyar a las autoridades nacionales y metropolitanas, o para paliar sus lagunas, así como también los problemas resultantes de la falta de eficacia y de coordinación (Dynes et al., 2010). Dentro de un contexto marcado por la debilidad inicial del Estado haitiano, la crisis provocada por el sismo de 2010 presencia la intervención de una cantidad importante de organismos internacionales y de ONG cuando las autoridades nacionales estaban rebasadas por la situación, planteando la cuestión institucional en el corazón de la crisis. En Nueva Orleans, las rivalidades entre las estructuras del poder contribuyeron ampliamente a amplificar la crisis, traduciéndose en conflictos entre las autoridades locales, la Guardia Nacional y las fuerzas de intervención federales dirigidas por la Secretaría de Defensa y el DHS (Department of Homeland Security) (Huret, 2010). Esta desorganización institucional ilustra los desafíos políticos y las cuestiones de competencias territoriales que cristaliza la gestión de crisis. 


\section{Frente a los desafíos de la crisis, diferentes formas de respuesta}

Frente a problemas que escapan a las normas y a situaciones de gran complejidad que rebasan las capacidades de gestión de las autoridades y rompen con el funcionamiento usual, las respuestas que vienen a paliar el desborde de los dispositivos oficiales son a menudo improvisadas.

Una primera forma de respuesta se basa en la intervención de las autoridades locales y de las poblaciones. Durante los tres primeros días siguientes al sismo de México D.F., la respuesta inmediata es esencialmente asumida por la población de las zonas afectadas, antes de que haya podido implementarse una coordinación a escala metropolitana. Los retornos de experiencia en Haití también han demostrado que, inmediatamente después del evento, la población misma extrajo a la gran mayoría de personas que permanecían vivas debajo de los escombros. Sin embargo, esta respuesta inmediata y local resulta siendo limitada en términos de eficacia y choca rápidamente con problemas de mayor envergadura que requieren medios más importantes.

Esta respuesta desordenada a escala local es seguida de una toma de control tardía y con frecuencia conflictiva por parte de las autoridades superiores: surgen desacuerdos en cuanto a las prioridades de acción, entre lo local y lo nacional, pero también entre autoridades públicas y actores privados. Estos últimos, con tiempos de reacción a menudo más rápidos, dan preferencia al funcionamiento y a la protección de sus propias infraestructuras, antes de tomar en consideración el requerimiento de las autoridades y el interés general (Guilhou et al., 2006). Finalmente, frente a perturbaciones de todo tipo, las modalidades normales del funcionamiento de la ciudad desaparecen dejando lugar a un funcionamiento extraordinario, alternativo y basado en la emergencia. Frente a la incapacidad del Estado, fueron las organizaciones internacionales y de ayuda humanitaria que asumieron la gestión de crisis en Haití.

\section{¿Cuáles son las dimensiones espaciales y territoriales?}

Más allá de las sorpresas específicas, que se multiplican al infinito (...), existe la sorpresa esencial: súbitamente el medioambiente está irreconocible. Tras Katrina, muchos habitantes explican que ya no reconocen su entorno geográfico, y que se pierden en barrios que antes les eran familiares (Guilhou et al., 2006:17).

Este ejemplo destaca un punto que interesa específicamente al geógrafo: las crisis plantean problemas espaciales y territoriales. Los espacios que registran directamente los daños constituyen un primer tipo de espacios producidos por la crisis, a imagen de los barrios centrales de México D.F. destruidos en más de $80 \%$ mientras la periferia casi no fue afectada. Otros espacios pueden dibujarse de manera indirecta. Los mapas producidos por los organismos internacionales para ayudar en la toma de decisiones luego del sismo de Haití muestran por ejemplo las zonas de agrupación espontánea de la población, los espacios aislados, los ejes de transportes y los puentes parcial o totalmente bloqueados por los escombros. Encontramos también estos espacios de la crisis en Nueva Orleans, tras las inundaciones que ocasionan el aislamiento de algunos sectores e imponen el uso de medios aéreos para resolver los problemas de desplazamientos. En Haití, al estar cortadas las carreteras, la distribución de la ayuda humanitaria tuvo que ser hecha por helicóptero en varias ocasiones.
Frente a los daños y a las dificultades de accesibilidad, se dibuja una nueva organización del espacio y una modificación de los usos del suelo. Así, asistimos a la reubicación de los lugares del poder de Puerto Príncipe del centro de la ciudad hacia el aeropuerto, el mismo que se constituye en cuartel general de las organizaciones nacionales e internacionales. Los campos de desplazados, al ocupar los parques, las plazas y los intersticios urbanos o lugares periféricos abandonados, trastocan la estructura urbana y generan nuevos desafíos de gestión.

Los lugares donde se encuentran los recursos que permiten manejar la situación de crisis se convierten así en nuevos espacios de polaridad, dando origen a flujos de bienes y de personas. En el campo de la salud, las transferencias de pacientes - desde las zonas afectadas del centro de México D. F. a los hospitales aún funcionales del resto de la ciudad; hacia otros Estados en el caso de Nueva Orleans; e incluso hacia otros países en el caso de Haití (hacia República Dominicana)- constituyen muchos de los fenómenos provocados por las crisis.

Algunas respuestas a los problemas planteados por las catástrofes se inscriben en cuanto a ellas en lógicas territoriales. Podemos destacar dos lógicas. En el primer caso, las acciones son efectuadas por actores locales a escala de territorios existentes (jurisdicciones de actores con competencias definidas). Las comunidades locales, las asociaciones de barrios y otras redes sociales, asumen la respuesta inmediata sobre su territorio usual, tal como ha sido el caso en México D. F. o en Haití.

La segunda forma de gestión corresponde a la creación de territorios ad hoc para enfrentar la crisis. El caso de Haití es revelador en este campo, en razón de la intervención de una cantidad importante de actores nuevos y de la escasa capacidad de respuesta de las autoridades locales. Los territorios usuales ya no son referencia y se instalan nuevos territorios de intervención, en función de los campos de acción de los nuevos actores, como las ONG o las fuerzas militares de apoyo.

Las crisis urbanas plantean pues una serie de problemas y de desafíos, tanto en términos de gestión como de análisis. Al tocar los diferentes aspectos precedentemente evidenciados, la cuestión de las dimensiones espaciales y territoriales es esencial. Ahora bien, no existen trabajos científicos ni operacionales que aborden este tema frontalmente.

\section{¿CÓMO SE ABORDA LA GESTIÓN DE CRISIS EN EL CAMPO DE LA GEOGRAFÍA?}

La cuestión de la gestión de crisis, pariente pobre de la investigación sobre los riesgos, es hoy insoslayable. El auge de la preparación para la gestión de crisis (preparedness) como nuevo paradigma de gestión de riesgos se traduce por la multiplicación de las iniciativas que van en este sentido y por la evolución de las temáticas de investigaciones. Sin embargo, para tratar la cuestión de las crisis desde el punto de vista de la geografía, hace falta construir un marco conceptual.

\section{El protagonismo de la preparación para la gestión de crisis}

Desarrollar las capacidades de gestión de crisis es un complemento necesario para las acciones de prevención, cuyos límites son por otro lado, ampliamente reconocidos. Este punto de vista ha sido reforzado, por los eventos que marcan el inicio de los años 2000, en particular el tsunami de 2004 en el Sudeste asiático, seguido del 
huracán Katrina en Nueva Orleans en 2005, y que contribuyen a la emergencia de una demanda social de información y de seguridad cada vez más fuerte por parte de la sociedad civil (Donze, 2007). El redoblado interés por las problemáticas de la gestión de crisis y por las medidas de protección civil responde a una doble preocupación: la primera se refiere al reconocimiento de la imposibilidad de eliminar riesgos, la segunda corresponde a la necesidad de actuar en una situación de incertidumbre que atañe tanto a las amenazas como a sus potenciales efectos. Una cosa es cierta, una catástrofe es posible y de lo que se trata es de prepararse para ella.

Antes considerada como un último recurso frente a la falta de prevención, la gestión de las crisis impone progresivamente su protagonismo, en el corazón de la gestión de los riesgos (NRC, 2006). Este avance de la crisis en el pensamiento sobre el riesgo rompe con el enfoque hasta ahora limitado a aspectos técnicos y logísticos, campo reservado tan sólo a los especialistas en seguridad civil. Este interés por la crisis va acompañado de la emergencia de un corpus conceptual en torno a la gestión de las crisis y a la preparación para las catástrofes, en particular la multiplicación de usos de la noción de resiliencia. El refuerzo de la seguridad civil y la preparación para el manejo de las situaciones de crisis, "disaster preparedness" pasan a ser entonces una prioridad, particularmente en el marco de Hyogo 2005-2015, y los enfoques preventivos pasan a un segundo plano.

\section{La gestión de crisis en la geografía}

Aunque el interés por un enfoque espacial de la gestión de las crisis sea reconocido (a menudo implícitamente), sin embargo esta cuestión sigue siendo todavía muy poco explorada por los investigadores, y en particular por los geógrafos. En efecto, las crisis no han sido nunca consideradas verdaderamente como objetos de investigación en geografía como lo pueden ser para otras ciencias sociales como la historia, la sociología o la antropología ${ }^{2}$. No obstante, la problemática ha sido tratada en cierto número de trabajos, más o menos directamente y desde diferentes ángulos de enfoque.

La información geográfica en el centro de la crisis: en respuesta a las crecientes necesidades de los actores operacionales de la gestión de crisis y gracias a las posibilidades de las nuevas tecnologías de la información y de la comunicación (NTIC), la información geográfica es cada vez más frecuentemente utilizada en la gestión de crisis. Esta tendencia es observable en los trabajos científicos (Curtis y Mills, 2010), pero también en los medios de comunicación, en particular en el tratamiento de los terremotos de Haití en 2010 y del Japón en 2011. El espectacular desarrollo de Open Street Map (http://openstreetmap.org) en Haití, para paliar la falta general de información geográfica, ilustra la imperiosa necesidad de este tipo de datos en periodo de crisis. Esto tiene que ser relacionado con el desarrollo de herramientas cada vez más sofisticadas, utilizadas por ejemplo para la realización de escenarios, desarrollo de programas informáticos de simulación, o también para la evaluación de los daños y la toma de decisiones. Los productos siguen siendo esencialmente técnicos y a menudo están focalizados sobre los fenómenos físicos. Se trata de las cartografías en tiempo real de las zonas afectadas, de las imágenes y fotos aéreas que permiten una comparación antes-después, de los sistemas de información geográfica en línea sobre una base participativa, de las animaciones y de los mapas interactivos, etc. Sin embargo, las dimensiones geográficas de las crisis no son abordadas directamente.
La crisis en los estudios de riesgo: numerosos trabajos clasificados como estudios del "riesgo", abordan más o menos directamente la cuestión de las crisis. Desde el punto de vista científico, los límites son vagos, y los resultados pueden ser a menudo útiles según las dos perspectivas del riesgo y de la crisis (la primera es ampliamente preferida). Definiendo al riesgo como una probabilidad, numerosos estudios de riesgo se basan en proyecciones o hipótesis: se analiza la vulnerabilidad y las pérdidas potenciales frente a un evento (una crisis) por venir (y respaldándose a veces sobre el análisis de crisis pasadas). Es el caso particularmente de los análisis costo-beneficio, en el que el ejercicio consiste en estimar daños potenciales a partir de uno (o varios) escenario(s) en relación con el costo de la prevención. Otras investigaciones ponen el acento sobre las condiciones físicas (exposición a las amenazas) y socioeconómicas (pobreza) capaces de llevar a una situación de crisis. Las capacidades de gestión de crisis (o la ausencia de capacidad) son tomadas en cuenta a veces para caracterizar la vulnerabilidad, así como la percepción del riesgo que puede influir sobre los comportamientos humanos en periodo de emergencia. A contrario, los procedimientos a posteriori tienen por objetivo analizar los riesgos y las vulnerabilidades basándose en eventos pasados. Estos se focalizan sobre los daños causados sin hacer de la gestión de crisis su objeto de investigación.

Los retornos de experiencias y análisis de crisis pasadas: la cuestión de la crisis es abordada de un poco más de cerca a través de los retornos de experiencias, que son verdaderos procedimientos institucionales sin por ello constituirse un objeto de investigación. Por otro lado, las grandes catástrofes y las crisis pasadas siempre han suscitado el interés de los geógrafos, a través por ejemplo del análisis de los daños y su distribución espacial o los límites de los sistemas de alertas y de las medidas de preparación. Otros se interesan más bien en las causas que origina las catástrofes o también en las incidencias sociales y territoriales de la gestión de las crisis. La catástrofe sirve entonces de soporte para analizar el antes y/o el después, las causas y/o los efectos.

Enfoques puntuales: movilidad y accesibilidad, comportamientos. Las dimensiones espaciales de la gestión de crisis han sido abordadas según aspectos muy específicos. Uno de ellos se refiere a las cuestiones de movilidad y de accesibilidad, apuntando hacia prever y anticipar las consecuencias de una perturbación de las redes de transporte, de aislamiento de territorios o de parálisis urbanas (Demoraes, 2004; Gleyze, 2005). Por ejemplo, algunos proponen herramientas operacionales, para la gestión de accidentes vinculados al transporte de materiales peligrosos (Griot, 2007). Las conductas humanas en situación de crisis también han atraído el interés de los geógrafos, por ejemplo, desde el ángulo de la conducta de las muchedumbres (Ruin, 2007; Provitolo, 2005), de la toma de decisiones, del tema de las alertas o también de las dificultades institucionales.

Enfoques más globales: desafíos de la gestión de crisis en las grandes aglomeraciones. Unos cuantos investigadores han abordado frontalmente la dimensión espacial de la gestión de crisis, en particular en el caso de investigaciones sobre los riesgos en el medio urbano. Es el caso de R. D'Ercole y de P. Metzger (2004) a través de una investigación exploratoria sobre la ciudad de Quito (Ecuador), en la que se identifican y localizan los desafíos mayores de la aglomeración, su vulnerabilidad y sus capacidades de enfrentar una crisis. El análisis de la vulnerabilidad de los sistemas urbanos se encuentra en los trabajos de C. Lutoff sobre Niza (2000) y 
de M. Reghezza sobre París (2006). Aunque no abordan directamente la gestión de crisis, estos aportan elementos de reflexión. En estas tres investigaciones, los desafíos del funcionamiento urbano son colocados en el centro del análisis del riesgo, con el objetivo de identificar disfuncionamientos, perturbaciones, daños o la pérdida potencial de algunos elementos que tienen repercusiones sobre el conjunto del sistema urbano. Este enfoque se asemeja además a aquel desarrollado en los Estados Unidos, en particular tras los acontecimientos del 11 de setiembre de 2001, que busca proteger las infraestructuras críticas a escala nacional. Aunque no formulado así, se trata en cierto modo de identificar los problemas de una gestión de crisis potencial a través de los fenómenos espaciales.

\section{Un marco conceptual original}

La problemática de la gestión de crisis es una de las preocupaciones del programa PACIVUR (Programa andino de formación y de investigación sobre los riesgos y las vulnerabilidades en el medio urbano) ${ }^{3}$. Las investigaciones efectuadas en el marco de este programa en las capitales andinas se han esforzado por abordar frontalmente la cuestión de las dimensiones espaciales y territoriales del manejo de situaciones de crisis. Estas han dado como resultado la formulación de un marco conceptual y una manera de proceder originales, con el objetivo de producir conocimientos sobre las vulnerabilidades del territorio en situación de crisis a partir del análisis de sus dimensiones geográficas.

El procedimiento propuesto parte de la hipótesis siguiente: desde el punto de vista espacial y territorial, el manejo de una situación de crisis consiste en poner en relación espacios afectados en el momento de la catástrofe (la demanda) por un lado, y por otro lado, recursos que permitan hacer frente a ello. Dentro de esta lógica, existen por lo menos dos tipos de espacios diferentes construidos por el manejo de las situaciones de crisis: por un lado los espacios directamente impactados por la catástrofe, a los que hay que prestar auxilio en forma prioritaria, y por otro lado los "espacios recursos" de gestión de crisis, es decir aquellos que disponen de los medios materiales y decisionales de auxilio y de recuperación. Desde el punto de vista espacial y territorial, la gestión de la crisis consiste en poner en relación a estos dos tipos de espacios (Cf. figura).

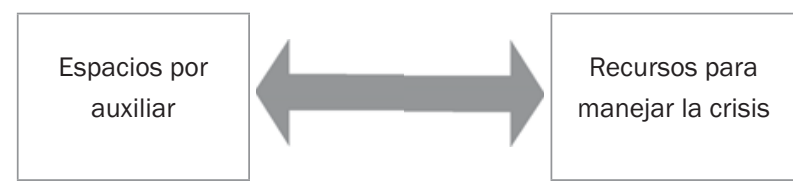

> Figura: Dimensiones espaciales del manejo de las situaciones de crisis.

En lo que respecta al primer tipo de espacio -los espacios por auxiliar - se considera a priori los espacios más vulnerables, en consecuencia los más susceptibles de ser afectados. Se trata en particular de espacios que concentran a una población vulnerable desde un punto de vista socio-económico, los que están altamente expuestos a las amenazas o también aquellos que presentan dificultades de accesibilidad.

La identificación de los recursos de la gestión de crisis supone proyectarse en una situación inédita que rompe con el funcionamiento usual del sistema urbano. Si bien una parte importante de los recursos que permiten manejar la crisis son elementos esenciales del funcionamiento normal (por ejemplo los principales hospitales de la aglomeración), algunos son elementos específicos de las si- tuaciones de crisis (los hospitales de campaña). También lo son las fuerzas armadas para el mantenimiento del orden o los camiones cisterna para el abastecimiento en agua potable en caso de ruptura de la red. La movilización de estos recursos específicos es significativa del paso a una situación de crisis, y ocurre cuando las capacidades del funcionamiento normal han sido rebasadas y es necesario recurrir a medidas excepcionales.

La puesta en relación de estos dos tipos de espacios es de varios órdenes. Por ejemplo, son relaciones de proximidad entre los establecimientos de cuidados de la salud y la población, pero también relaciones de dependencias y de capacidades de acceso. El análisis de estas relaciones permite calificar los territorios. De hecho, las dificultades de adecuación, ya sean físicas o funcionales entre la demanda y los recursos, contribuyen a la vulnerabilidad del sistema de gestión de crisis y por ende a la vulnerabilidad del territorio en caso de crisis.

Este enfoque de la gestión de crisis tiene por objetivo delimitar las dimensiones espaciales y territoriales que las caracterizan. Busca producir conocimientos sobre las vulnerabilidades del territorio, a partir del análisis de crisis pasadas o en la perspectiva de una crisis venidera.

\section{LA CRISIS COMO OBJETO DE INVESTIGACIÓN DE LA GEOGRAFÍA}

Se abren nuevas pistas de reflexión al considerar la gestión de crisis como un objeto de investigación de la geografía. Tomar en cuenta las dimensiones espaciales de la gestión de crisis permite en efecto enriquecer los enfoques más clásicos sobre los riesgos. Este procedimiento implica también una reflexión sobre los procesos sociales y territoriales de construcción de la vulnerabilidad que originan las crisis.

\section{Proyectarse para una crisis mayor}

Proyectarse para una crisis mayor impone superar la definición clásica de los riesgos y pensar el funcionamiento urbano en su conjunto. Una crisis mayor es interesante de analizar justamente porque cuestiona el funcionamiento urbano, evidenciando múltiples interrelaciones y dependencias.

Los problemas ocasionados por eventos circunscritos no se pueden comparar con aquellos de una crisis mayor. Estos últimos no son solamente más importantes desde el punto de vista cuantitativo (más muertos y heridos, etc.), sino sobre todo porque se suman a problemas de otra índole: ruptura del abastecimiento en agua, en energía, en alimentos, daños y saturación de los establecimientos de cuidados de la salud, pérdida de las telecomunicaciones, o también amenazas a la salvaguarda del orden público. Los territorios afectados son también más extensos, superando ampliamente los lugares del impacto inicial. Proyectarse para una crisis implica hacer estas hipótesis - pensar lo impensable- como lo preconiza P. Lagadec. Se trata de pensar el disfuncionamiento generalizado de los servicios urbanos y de la ciudad en general. Estos disfuncionamientos pueden ser ocasionados directamente por una perturbación exterior, pero también a partir de vulnerabilidades propias del funcionamiento urbano. En efecto, si la amenaza es el estímulo, este representa una forma de vulnerabilidad entre otras que juntas contribuyen a generación de un situación de crisis. Proyectarse para una crisis mayor permite revelar sus vulnerabilidades. Esta constituye de hecho un punto de entrada y una clave de lectura, 
porque impone imaginar los disfuncionamientos en todas las es calas del territorio, en un contexto de incertidumbre generalizada.

\section{Crisis y transmisión de vulnerabilidad}

La perspectiva de una crisis mayor impone pensar los disfuncionamientos urbanos y sus consecuencias. Estos disfuncionamientos tienen múltiples orígenes e ilustran las interrelaciones no sólo entre diferentes tipos de recursos, sino también entre los recursos y el territorio.

El funcionamiento de un recurso particular depende necesariamente de varios otros recursos. Por ejemplo, el buen funcionamiento de los hospitales depende de los otros servicios urbanos tales como el abastecimiento en agua, en energía o también de los sistemas de telecomunicaciones. La transmisión de vulnerabilidad de los servicios urbanos hacia los hospitales puede estar limitada cuando estos últimos disponen de alternativas y/o de una autonomía suficiente. Por otro lado, los recursos de gestión de crisis responden a algunas necesidades y su disfuncionamiento puede afectar todo o parte del territorio urbano. El daño de un hospital mayor a escala de una ciudad puede provocar la pérdida del acceso a los servicios de salud para una gran cantidad de personas. En retorno, un gran número de pacientes puede provocar la saturación de un hospital e impidiendo su buen funcionamiento.

Una visión territorial de la gestión de crisis consiste pues en tomar en consideración no solamente la adecuación entre la oferta y la demanda, sino sus interrelaciones, incluso hasta sus retroacciones. La preparación para la gestión de crisis debe integrar por un lado la interconexión y la interdependencia de las diferentes redes, infraestructuras y equipos, y por otro lado las relaciones entre la oferta y la demanda sobre el territorio. Reflexionar a partir de los recursos permite identificar los fenómenos de transmisión de vulnerabilidades, y de elaborar estrategias para interrumpir los efectos en cadena. La gestión de crisis implica pensar el funcionamiento del territorio en su conjunto.

\section{Pensar un continuum "riesgo-crisis"}

La lógica del auxilio es diferente de la de prevención de los riesgos:

Dos perspectivas se oponen aquí: aquella que consiste en privilegiar el salvataje de vidas en el momento del evento y la que insiste sobre las profundas transformaciones que hay que hacer fuera del evento en sí. Y estas dos perspectivas remiten a instituciones, competencias y maneras de pensar situaciones muy diferentes (S. Revet, 2011).

Analizar la vulnerabilidad del territorio en caso de crisis supone considerar las crisis como una construcción social y territorial. Requiere el análisis de situaciones contingentes y de las respuestas a los desastres, interpretándolas al mismo tiempo en el marco de una visión social, política e histórica de los riesgos, fuera del evento en sí. En efecto, las vulnerabilidades que contribuyen a la emergencia de una crisis resultan de un conjunto de problemas de fondo: la pobreza, la calidad de los servicios urbanos, la ausencia de política de planificación preventiva de los riesgos, por no citar sino algunos. Estas vulnerabilidades van a alimentar la crisis venidera: El evento entra en resonancia con su contexto. Como un ciclón que acrecienta su fuerza extrayendo su energía de los mares que atraviesa, este se va a alimentar de todos los problemas y desequilibrios ya presentes dentro del contexto donde se mueve (Lagadec, 1991). No se pueden pues analizar las crisis venideras haciendo abstrac- ción de los procesos a largo plazo que contribuyen a construirlos. En retorno, las crisis pueden ser tomadas como indicadores de vulnerabilidad, en el sentido en que estas exacerban y revelan las vulnerabilidades del territorio.

El enfoque geográfico de la gestión de crisis trata de evidenciar los vínculos entre las lógicas de fondo y los problemas potenciales en el momento de la crisis, y de qué manera estos vínculos se materializan sobre los territorios urbanos. La crisis no es entonces vista como una fase más o menos independiente de la gestión (integral) de los riesgos, sino que se inscribe en un continuum prevención de los riesgos-preparación y gestión de crisis. Pensar este continuum implica reformular los problemas, explicitar los vínculos entre prevención y preparación, pensar a la vez los procesos de construcción de vulnerabilidades en el tiempo largo y las transmisiones de vulnerabilidades en el seno del territorio.

De hecho, las medidas de prevención de los riesgos mejoran necesariamente la preparación para las crisis: reforzar un hospital o mejor aún planificar la oferta de servicios de salud sobre el territorio reducen las vulnerabilidades capaces de alimentar la crisis. Inversamente, las acciones de preparación mejoran el funcionamiento urbano y son capaces de disminuir las vulnerabilidades de fondo: reforzar las coordinaciones entre actores, y la autonomía de los servicios básicos de los establecimientos de cuidados de la salud o también implicar las redes sociales y la participación ciudadana. De cierta manera se trata de socializar la preparación a la gestión de crisis y de integrarla en las políticas urbanas.

Pensar un objeto científico construido en un continuum "riesgocrisis" implica superar las fragmentaciones temporales impuestas por la acción -el antes, el durante, el después-. Se trata también de incorporar la problemática de los riesgos y de las crisis a la del funcionamiento urbano y del desarrollo en general. En efecto, los procesos de construcción de los riesgos y de las crisis son indisociables de las modalidades de producción de la ciudad, y por eso mismo del desarrollo. También, proyectarse para una situación de crisis mayor - una crisis del territorio urbano- permite leer conjuntamente las problemáticas de los riesgos y de la ciudad.

\section{POR UNA GEOGRAFÍA DE LA GESTIÓN DE CRISIS}

El análisis de las dimensiones espaciales y territoriales de la gestión de las situaciones de crisis ofrece una mirada nueva y abre perspectivas de investigaciones sobre la problemática de los riesgos y de las crisis. El enfoque propuesto presenta primero el interés de proponer un marco conceptual y un procedimiento que permiten producir conocimientos sobre las situaciones de crisis, sean pasadas o venideras. Trata también de revelar las vulnerabilidades de los territorios, superando los enfoques clásicos en términos de riesgos, a menudo focalizados sobre el estudio de las amenazas y a escala local. El enfoque propuesto se esfuerza así por contribuir a la mejora de la comprensión de las vulnerabilidades urbanas dándoles (o volviendo a darles) toda su profundidad política y social. El análisis de las dimensiones espaciales y territoriales de la gestión de crisis permite comprender a la vez el funcionamiento urbano y los procesos de construcción del territorio. Las crisis vistas desde el ángulo de la geografía son claves de lectura de lo social y de lo político. El territorio, como espacio de funcionamiento y de poderes, intrínsicamente político, está en el centro de esta geografía. 


\section{BIBLIOGRAFÍA}

CURTIS A., MILLS J. W. (2010) GIS, Human Geography, and Disasters, Cognella, Donze J., 2007, Le risque: de la recherche à la gestion territorialisée, Géocarrefour, Vol. 82/1-2, 2007, http://goecarrefour.revues.org/1395

DEMORAES F. (2004) Mobilité, enjeux et risques dans le District Métropolitain de Quito (Équateur), Tesis de doctorado en geografía, Universidad de Saboya, Chambéry.

D'ERCOLE R., METZGER P. (2004) Vulnerabilidad del Distrito Metropolitano de Quito, Colección Quito Metropolitano, MDMQ-IRD, Quito, Ecuador.

DUBOIS-MAURY J., CHALINE C. (2002) Les risques urbains, Armand Colin, París.

DYNES R. R., QUARANTELLI E. L., WENGER D. (1990) Individual and organizational response to the 1985 earthquake in Mexico City, Mexico, University of Delaware, Disaster Research Center, Book \& Monograph series \# 24, http:// dspace.udel.edu:8080/dspace/handle/19716/2259

GLEYZE J. F. (2005) La vulnérabilité structurelle des réseaux de transport dans un contexte de risques, Tesis de doctorado, Universidad de París VII. Denis Diderot.

GRIOT C. (2007) Vulnérabilité et transport de matières dangereuses: une méthode d'aide à la decisión issue de l'expertise de la Sécurité Civile, Cybergeo: Revue européenne de géographie, №361, http://www.cybergeo.eu/ docannexe/file/5995/361.pdf

GUILHOU X., LAGADEC P., LAGADEC E. (2006) Les crises hors cadres et les grands réseaux vitaux-Katrina. Faits marquants, pistes de réflexion. EDF, Direction des Risques Groupe, avril 2006.

HURET R. (2010) Katrina, 2005. L'ouragan, l'État et les pauvres aux États-Unis, París: Édition EHESS, Cas de Figure, 2010.

LAGADEC P. (1991) La Gestions des Crises. Outils de réflexion à l'usage des décideurs, París: McGraw Hill- Ediscience.

LUTOFF C. (2000) Le Systeme Urbain niçois face à un séisme: méthode d'analyse des enjeux et des dysfonctionnements potentiels, Tesis de doctorado, Universidad de Saboya, Chambéry.

MICHEL-KERJAN E. (2003) Risques catastrophiques et réseaux vitaux: de nouvelles vulnérabilités, Flux 2003/1, N 51, páginas 6-15.

NRC (2006) Facing Hazards and disaters, Understanding Human Dimensions, Committee on Disaster Research in the Social Sciences: Future Challenges and Opportunities, National Research Council - NRC.

PROVITOLO D. (2005) Un exemple d'effets de dominos: la panique dans les catastrophes urbaines, Cybergeo, Systèmes, Modélisation, Géostatiques, $N^{\circ} 3$, http://www. cubergeo.eu/index2991.html

REGHEZZA M. (2006) Réflexions autour de la vulnérabilité métropolitaine: la métropole parisienne face au risque de crue centennale, Tesis de doctorado, Universidad París X-Nanterre.
REVET S. (2011) "Penser et affronter les désastres: un panorama des recherches en sciences sociales et des politiques internationales", Critique internationale, 2011/3 No 52 , páginas 157-173.

ROBERT, J. (2012), Pour une géographie de la gestion de crise: de l'accesibilité aux soins d'urgence à la vulnérabilité du territoire à Lima, Tesis de geografía de la Universidad de Grenoble, http://tel.archives-ouvertes.fr/tel-00766252

RUIN, I. (2007) Conduite à contre courant. Les pratiques de mobilité dans le Gard: facteur de vulnérabilité aux crues rapides, Tesis de doctorado, Universidad Joseph Fourier.

\section{NOTAS}

1 Este artículo es una traducción de un texto publicado en francés en la revista de l'Institut National des Hautes Etudes de la Sécurité et de la Justice. ROBERT J. (2013) La gestion de crise: un point de vue de géographe, Cahiers de la Sécurité $\mathrm{N}^{\circ}$ 24 "2013. Affronter les crises. Vingt ans de réflexions", Revue de I'Institut National des Hautes Etudes de la Sécurité et de la Justice. Juin 2013, páginas 38-46, http://www.inhesj.fr/fr/quisommesnous/les- publications/les-cahiers-de-la-securite/24

2 Véase especialmente los trabajos de P. Lagadec en Francia y de E. Quarantelli sobre la sociología de los desastres en los Estados Unidos.

3 PACIVUR es un programa regional del IRD (Instituto de Investigación para el Desarrollo) en Bolivia, Ecuador y Perú desde el año 2006. http://www.prodig.cnrs.fr/spip.php?article1885

38 > Revista Márgenes № 15 Vol 11 > Diciembre 2014: 32 a 38 Facultad de Arquitectura > Universidad de Valparaíso 